\title{
Understanding patterns of brain metastasis in breast cancer and designing rational therapeutic strategies
}

\author{
Evelyn M. Brosnan, Carey K. Anders \\ Division of Hematology/Oncology, Lineberger Comprehensive Cancer Center, University of North Carolina, Chapel Hill, NC, USA \\ Contributions: (I) Conception and design: All authors; (II) Administrative support: All authors; (III) Provision of study materials or patients: All \\ authors; (IV) Collection and assembly of data: All authors; (V) Data analysis and interpretation: All authors; (VI) Manuscript writing: All authors; (VII) \\ Final approval of manuscript: All authors. \\ Correspondence to: Carey K. Anders, MD. Division of Hematology/Oncology, Lineberger Comprehensive Cancer Center, University of North \\ Carolina, 170 Manning Drive, Campus Box 7305, Chapel Hill, NC 27599-7305, USA. Email: carey_anders@med.unc.edu.
}

\begin{abstract}
One of the most feared sequelae after a diagnosis of advanced breast cancer is development of metastases to the brain as this diagnosis can affect physical function, independence, relationships, quality of life, personality, and ultimately one's sense of self. The propensity to develop breast cancer brain metastases (BCBMs) varies by subtype, occurring in up to one half of those with triple negative breast cancer (TNBC), approximately a third of HER+ breast cancers and $14 \%$ in hormone positive disease. Median survival after BCBM diagnosis can be as short as 5 months in TNBC and 10-18 months in the other subtypes. Here, we review the biology of BCBMs and how it informs the rational design of new therapeutic approaches and agents. We discuss application of novel targeted and immunotherapies by breast cancer subtype. It is noteworthy that there are no U.S. Food and Drug Administration (FDA)-approved treatments specifically for BCBMs currently. Nevertheless, there are legitimate grounds for hope as patients with BCBMs are now being included in clinical trials of systemic therapies and a better understanding of the biology and genetic underpinning of BCBMs is driving an increased range of options for patients.
\end{abstract}

Keywords: Breast cancer; brain metastases; genomic; immunotherapy; targeted therapy

Submitted Dec 15, 2017. Accepted for publication Mar 06, 2018.

doi: $10.21037 / \mathrm{atm} .2018 .04 .35$

View this article at: http://dx.doi.org/10.21037/atm.2018.04.35

\section{Introduction}

A diagnosis of brain metastases is a devastating consequence of advanced breast cancer. "Your brain controls your independence, your quality of life, your entire existence," one woman wrote. "Brain metastases can bring on a loss of hope and a fear of loss of self. It's not just a body part that's at risk, it's our life as who we are." (1).

Overall, $10-30 \%$ of patients with metastatic breast cancer (MBC) with develop brain metastases during the course of their disease (2-4). However, as is the case in primary breast cancers, subtype is paramount to metastatic behavior and overall survival. For hormone positive disease [estrogen (ER) + and/or progesterone receptor (PR)+], the incidence of breast cancer brain metastases (BCBMs) is 14\% (5), with a median overall survival after the development of brain metastases of $9-10$ months (5-7). About $34 \%$ of patients with HER2 + breast cancer will develop CNS metastases, however median survival following central nervous system (CNS) metastasis is approximately 11-18 months (5-8). Using data from the HERA trial, Pestalozzi et al. reported that adjuvant HER2-directed therapy with trastuzumab did not increase the risk of CNS metastasis. The incidence of CNS relapse as a first DFS event was $2 \%$ in both the trastuzumab and observation only arms. Moreover, the cumulative incidence of CNS relapse during the entire course of the disease was higher in the observation group (57\%) compared to those who received 1 year of trastuzumab (47\%), $\mathrm{P}=0.06$ (9). The incidence of CNS metastases is as high was $46 \%$ among patients with 
advanced triple negative breast cancer (TNBC), with 14\% having CNS metastasis at initial diagnosis of metastatic disease (10). Median survival from the diagnosis of triple negative CNS metastases is only 4.9 months $(6,10)$.

Complicating matters is the fact that discordance between the intrinsic molecular subtype of the primary breast cancer and matched brain metastasis can occur up to $20 \%$ of time, predominantly loss of hormone receptor expression (ER or PR) and gain of HER2 overexpression $(11,12)$. This is especially challenging when selecting systemic therapies as access to CNS tissues are limited; surgical resection of CNS metastases is typically limited to solitary metastases, space occupying lesions, and in cases where the diagnosis is in question. As such, radiation therapy remains the predominant modality for treating BCBM. To date, there are no U.S. Food and Drug Administration (FDA)-approved systemic treatments for BCBM. In this review article, we will provide an overview of the current knowledge of blood brain/tumor barrier, the biology of brain metastases and inherent CNS microenvironment, systemic therapies (including novel delivery platforms and immunotherapy) in development for the treatment of BCBMs.

\section{The blood-brain barrier (BBB)}

The BBB exists to selectively regulate what enters the brain and protects it from toxic substances, including chemotherapeutics and targeted drugs. It is a complex barrier consisting of capillary endothelial cells (ECs) surrounded by a basement membrane, astrocyte foot processes covering $90 \%$ of the EC surface and pericytes $(13,14)$. Brain ECs are uniquely designed for this purpose by having features that severely limit passive diffusion—tight intracellular junctions, lack of fenestrations, low pinocytosis and high electrical resistivity $(14,15)$.

Beyond this physical barrier, the surrounding astrocytes and pericytes secrete a variety of molecules that affect transport, signaling, angiogenesis and include ecto- and endo-enzymes that degrade drug molecules, forming an enzymatic BBB to entry (16-19). In addition, ECs contain transporters such as $\mathrm{P}$-glycoprotein, multidrug-resistance proteins MDRP1-9, ABCG2 (the breast cancer resistance protein) and organic anion transporters (OATs), which act as efflux pumps, removing drugs and toxins from the brain.

Glucose and amino acids are actively transported across the BBB. Larger essential molecules enter by specific receptor-mediated endocytosis (e.g., insulin receptor, transferrin receptor for iron, lipoprotein receptor) (20). Some small molecules can passively diffuse across the $\mathrm{BBB}$, but only if they have certain key characteristics: molecular weight generally $<400-500$ daltons, lipophilicity, low hydrogen bonding ability $(14,21)$. Muldoon et al. (22) showed that in addition to size, uptake of a number of common chemotherapy drugs was compromised by significant plasma protein binding ( $90 \%$ or more) and being substrates for the efflux pumps (e.g., paclitaxel, vincristine, vinblastine, doxorubicin, etoposide). Inhibition of P-glycoprotein transport has been shown to increase brain levels of paclitaxel (23), and daunorubicin (24).

\section{Blood-brain vs. blood-tumor barrier in brain metastases}

Studies showing response in the brain to chemotherapy agents suggested that the defenses of the $B B B$ are disrupted in the presence of brain metastases. In 100 breast cancer patients with brain metastases, Rosner et al. (25) reported objective response in the brain in $50 \%$ of the patients treated with 3 or 5 drug combination chemotherapy (cyclophosphamide, 5-fluouracil, doxorubicin, methotrexate, vincristine). Subsequent studies describe compromised tight junctions, fenestrations in the vasculature and alterations in the expression of efflux transporters that make the BBB more permeable $(26,27)$.

In a preclinical study using human xenograft and murine breast cancer cell lines, Lockman et al. (28) examined over 2,000 brain metastases and found significant heterogeneity in BBB permeability and uptake of chemotherapy drugs between metastases affecting outcome. While $>89 \%$ of metastases showed increased BBB permeability compared to normal brain, this was unrelated to tumor size and varied both within and between metastases. Although the blood brain barrier was compromised, their data showed that there was still sufficient integrity to affect uptake and efficacy of ${ }^{14} \mathrm{C}$-paclitaxel and ${ }^{14} \mathrm{C}$-doxorubicin. Again there was heterogeneity in uptake within and between metastases, with up to a 200-fold difference and no correlation with tumor size. Concentrations of these drugs were higher in metastases than normal brain cells, but was still $<15 \%$ of that in peripheral metastases. Moreover only about $10 \%$ of the most permeable metastases reached cytotoxic levels of the drug.

Tasker et al. (29) had similar findings in a HER2 breast cancer preclinical model of CNS uptake of the lipophilic, small tyrosine kinase inhibitor (TKI) ${ }^{14} \mathrm{C}$-lapatinib. They 
found that distribution of the drug in brain metastases was again highly variable. ${ }^{14} \mathrm{C}$-lapatinib concentration was $<10-20 \%$ of that in peripheral metastases, with just a small subset $(17 \%)$ reaching comparable levels to extracranial metastases. Thus, the partially intact BBB was still capable of negatively affecting drug delivery.

In another murine preclinical breast cancer model, Adkins et al. (30) showed that the P-glycoprotein efflux pump function appears to remain intact in metastases and is comparable to that of the normal BBB. Interestingly, paclitaxel, doxorubicin and lapatinib in the previous studies are all substrates for P-glycoprotein transporter. In summary, the unpredictable nature and heterogeneity in permeability of the BBB, in addition to inherent drug efflux pumps, makes it challenging to effectively deliver drugs in sufficient quantity to brain metastases to achieve apoptosis.

\section{Biology of BCBMs}

Breast cancer cells must cross the BBB to establish metastases and the mechanisms by which they do so are the subject of continued study. Comparative genomewide expression analysis by Bos et al. (31) using a human umbilical vein endothelial cells (HUVECs) in vitro model of the BBB, identified genes that facilitate breach of the BBB: cyclooxygenase COX2 (increases BBB permeability by prostaglandin production), epidermal growth factor receptor (EGFR) ligand HBEGF (increases cancer cell motility and invasiveness) and alpha 2,6-sialyltransferase ST6GALNAC5 (enhanced breast cancer cell adhesion to brain ECs and enable transmigration). However, Drolez et al. (32), using a different human BBB in vitro model with $\mathrm{CD} 34^{+}$ derived ECs co-cultivated with brain pericytes called brain-like endothelial cells (BLECs), found the opposite effect with ST6GALNAC5 expression resulting in a decrease in adhesion of breast cancer cells to the BBB model and no change in BBB permeability. This speaks to the challenges of developing pre-clinical models that can accurately represent the mechanisms of in vivo human brain metastases.

In an in vivo mouse model, TNBC cells were shown to lodge in the capillaries and induce Angiopoeitin-2 expression in local brain ECs with decreased in Zonula occludens (ZO-1) and claudin-5, key scaffold/structural proteins in the tight junctions, resulting in a "leaky" BBB. Treatment with the Angiopoeitin-1/2 neutralizing peptibody, trebananib, prevented breakdown of the BBB and development of brain metastases (33).
Downregulation of efflux transporter proteins can also create a more permeable BBB. A small study of 29 resected brain lesions showed that brain metastases in TNBC were negatively correlated with the expression of the transporters GLUT1 (glucose transporter 1) and breast cancer-resistant protein ABCG2 (also known as BCRP) in intratumor capillaries, causing disruption of the BBB (34). By contrast, in Her2/neu+ breast cancer, the correlation was a positive one, leading to a possible conclusion that Her2/neu+ breast cancer cells migrate into the brain without a substantial breach of the BBB.

Alterations in the HER family receptors have be associated with brain colonization.

Expression of HER2 is enriched in brain metastases compared with primary tumors and in a preclinical model, transfection of HER2 into an inherently HER2-negative human breast cancer cell line resulted in a 3 -fold increase of large brain metastases, linking a gain of HER2 with colonization in the brain (35). HER3 overexpression and activation of downstream pathways (particularly the MAPK pathway) were increased in brain metastases compared to the primary tumor in human samples (36).

To explore genetic alterations underlying brain metastases, Sahlia et al. (37) performed genomic/epigenomic analysis on fresh frozen samples of breast brain metastases. Large gains in 1q, 5p, 8q (MYC), 11q, 20q and deletions in $8 \mathrm{p}, 17 \mathrm{p}, 21 \mathrm{p}, \mathrm{Xq}$ were observed in chromosomal analysis. Frequently amplified and overexpressed genes included ATAD2, BRAF, DERL1, DNMTRB, NEK2A. By contrast $A T M, C R Y A B, H S P B 2$ genes were deleted or underexpressed in brain metastases. Knowledge mining revealed enrichment in cell cycle and G2M transition pathways (i.e., AURKA, AURKB, FOXM1). DNA methylation was increased overall but notably, basal-like BCBMs exhibited significantly lower levels of methylation. Although a small study, ( $\mathrm{n}=35$ brain metastasis samples, $\mathrm{n}=10$ non-neoplastic brain and breast samples, $\mathrm{n}=50$ early stage breast cancer samples), it nevertheless highlights areas that may be fruitful for additional investigation to ultimately identify targetable alterations in brain metastases.

\section{Crosstalk between tumor and brain microenvironment}

Breast cancer cells also adapt to the local microenvironment in the brain and co-opt neurons, astrocytes and microglial cells to proliferate and establish metastases.

Metastatic breast cells have been shown to develop 
neuronal characteristics, expressing the $\mathrm{GABA}_{\mathrm{A}}$ receptor, GABA transporter, GABA transaminase, parvalbumin, and reelin, allowing them to take up GABA, shunt it to nicotinamide adenine dinucleotide phosphate (NADPH) production and facilitate proliferation of the tumor cells in the brain microenvironment (38). Kim et al. (39) showed that murine astrocytes co-cultured in direct cell-to-cell contact with human breast cancer cells caused up-regulation of survival genes (GSTA5, BCL2L1, and TWIST1) in the tumor cells, thus protecting them from the toxic effects of chemotherapy.

Zhang et al. (40) demonstrated that microRNAs from astrocytes cause human and mouse tumor cells with normal expression of PTEN, to downregulate PTEN expression in the brain environment. The loss of this tumor suppressor gene expression allows proliferation of brain metastases. Subsequent blockade of astrocyte secretion restored PTEN and suppressed brain metastasis in vivo. Loss of PTEN is associated TNBC subtype and portends a shorter survival time. Hohensee et al. (41) showed that upregulation of PTEN in a TNBC cell line led to reduced migration and invasion to the brain. Autocrine and paracrine activation of GM-CSF/CSF2RA and AKT/PTEN pathway on both astrocytes and tumor cells mediated this crosstalk.

A better understanding of immunogenicity of the tumor microenvironment in brain metastases may reveal a role for immunotherapy in treatment of BCBMs, though this is still in its infancy. Tumor infiltrating lymphocytes (TILs) are prognostic in TNBC with higher levels of TILs associated with lower distant metastases. Higher level of TILs also predicts increased trastuzumab benefit in HER2+ disease. The immune environment in brain metastases is less well characterized. Three abstracts presented at ASCO 2017 focused on this topic. Narloch et al. (42) compared 37 matched primary and brain metastatic tumors. The amount of TILs in the primary tumor did not predict time to brain metastases. However, percentage of TILs was markedly reduced in metastasis compared to primary, with the largest decrease seen in TNBC subtype. Ogiya et al. (43) also found higher levels of TILs in primary compared to metastatic breast tumors, The median category of TILs of BM tumors was $1-10 \%$ (range, $1-30 \%$ ). In their study, patients with TNBC with low TILs percentage had significantly shorter overall survival compared with those with high TILs ( $\mathrm{P}=0.04)$. Prince et al. (44) examined the prognostic significance of 4 biomarkers in the brain microenvironment in a biobank of 203 breast cancer patients-gliosis, immune infiltrate, hemorrhage and necrosis. Across all samples, expression of gliosis, immune infiltrate and hemorrhage was associated with increased overall survival, while necrosis was associated with inferior survival. Collectively, this data illustrates a crucial role for tumor and brain microenvironment interaction that provides a rich arena to identify new therapeutic options for BCBM, such as immunotherapy.

\section{Local therapies "by-passing" the BBB to treat metastases}

As yet, there are no approved breast-cancer-specific treatment modalities for BCBMs. Rather, the standard of care is local therapy for secondary brain metastases, in general, and uses techniques that essentially "by-pass" the $\mathrm{BBB}$ - surgical resection and radiation. The choice is determined by such factors as number, size and location of the brain lesions and performance status of the patient. While an exhaustive review is beyond the scope of this paper, generally and for a solitary lesion, treatment is surgical resection $+/$ - stereotactic radiosurgery (SRS), less commonly, +/- whole brain radiotherapy (WBRT) given longer term neurocognitive sequelae $(45,46)$. For limited metastases (1-4 lesions), SRS alone is preferred over WBRT or combination SRS/WBRT given no overall survival advantage and significant neurocognitive toxicity associated with WBRT (46-48). For multiple metastatic lesions ( $>4$ lesions) or where surgery or SRS is not feasible, WBRT is the primary therapy.

A variety of pharmacologic agents as radiosensitizers have been studied combined with WBRT to increase the efficacy of the radiation, but several meta-analyses $(49,50)$ concluded that they did not offer any overall survival advantage or increased tumor response compared to WBRT alone. Clinical trials are continuing, however, with newer agents such as lapatinib, (NCT01622868) and sorafenib (NCT01724606) as radiosensitizers for WBRT.

Other studies are exploring how to leverage the abscopal effect of RT, i.e., local irradiation inducing tumor regression at non-irradiated, distant tumor sites. The mechanism is thought to be via immunogenic cell death causing subsequent host immune stimulation to fight tumors. Accordingly, there is interest in combining RT with systemic immunotherapy checkpoint inhibition to enhance this effect. McArthur et al. studied 20 patients with $\mathrm{BCBMs}$ requiring treatment with standard of care WBRT or SRS and combined this with Tremelimumab immunotherapy—a monoclonal antibody against cytotoxic 
lymphocyte antigen-4 (CTLA-4) (51). For HER2-negative disease, the best response was 2 patients with stable nonCNS disease at 12 weeks but 2 of the HER $2+$ patients on concurrent trastuzumab therapy had partial response/ stable non-CNS disease which was durable at 6 months. A pilot study to evaluate for efficacy of brain Irradiation with tremelimumab +/- trastuzumab in BCBM is now underway (NCT02563925).

Hippocampal-sparing WBRT (HS-WBRT) has emerged as a novel way to protect against the neurocognitive toxicity of standard WBRT, preserving memory and quality of life (52). Wu et al. (53) studied 192 breast cancer patients with a total of 1,356 metastases and found only $3.6 \%$ had hippocampal metastases (HM) and $7.3 \%$ had perihippocampal metastases (PMH), i.e., hippocampus plus a $5-\mathrm{mm}$ margin, supporting this as a reasonable treatment approach given the low incidence of metastases in this region that may not be covered in the HS-WBRT field. In addition, they found that patients with $>10$ metastases were at higher risk of PHM. Although they found no association between breast cancer subtype and $\mathrm{PMH}$, those with $>10$ brain lesions in their study were in the HER2/neu and TNBC subtypes. A clinical trial (NCT03002532) is currently enrolling patients to compare neurocognitive function and prognosis between HS-WBRT and conventional WBRT for treatment of BCBMs.

A pharmacologic approach using the N-methyl-Daspartate (NMDA) receptor antagonist drug memantine has also been shown to reduce neurocognitive decline from WBRT. Brown et al. (54) found that memantine during and after WBRT resulted in delayed time to cognitive decline and reduced rates of decline in memory, executive functioning and processing speed. Accordingly, a clinical trial is now recruiting combining both HS-WBRT and memantine for neurocognitive protection (NCT02360215).

A very different physical approach that "by-passes" the challenges of the BBB uses tumor treating fields (TTFs). TTFs are low intensity $(1-2 \mathrm{~V} / \mathrm{cm})$, intermediate frequency $(100-200 \mathrm{kHz})$, alternating electric fields that induce unidirectional forces that interfere with spindle tubulin polymerization in cells undergoing mitosis, resulting in apoptosis $(55,56)$. Optune ${ }^{\circledR}$ is a wearable device (Novocure, St. Helier Jersey; NASDAQ: NVCR) that delivers TTFs via disposable ceramic transducers applied directly to the patient's scalp. In 2015, the FDA approved Optune ${ }^{\circledR}$ combined with temozolomide, in newly diagnosed glioblastoma multiforme (GBM) patients after surgery and chemoradiation given a 3 -month progression free and overall survival advantage for the combination compared to temozolomide alone (57). The device was previously approved in 2011 as a salvage monotherapy in patients with recurrent GBM. New studies are exploring application of TTF modality to metastatic disease in NSCLC - a phase II clinical trial of TTFs after standard local treatment in patients with 1-5 brain metastases (NCT01755624) and a Phase III trial (METIS) exploring the effects of TTFs in NSCLC patients with 1-10 brain metastases following radiosurgery (NCT02831959). This modality has not yet been applied specifically to BCBMs.

\section{Systemic therapies for BCBMs}

\section{HER2 positive disease}

The mainstay of systemic HER2-directed therapies rely on large monoclonal antibodies targeting HER2 or HER2/ HER3, i.e., trastuzumab, pertuzumab and the antibodydrug conjugate ado-trastuzumab emtansine (T-DM1), and are thus are incapable of crossing an intact BBB. However, impaired BBB at site of metastases $(26-29,58)$ and radiation (59) can precipitate a "leaky" BBB allowing these large molecules greater access. In a preclinical model using mouse transgenic breast tumor cells expressing human HER2 (Fo2-1282 or Fo5), Lewis Phillips et al. (60) established intracranial tumors by stereotactically injecting HER2 + tumor cells directly into striatum of mice brains. Systemic ${ }^{89} \mathrm{Zr}$-trastuzumab was administered and PET imaging revealed specific uptake of ${ }^{89} \mathrm{Zr}$-trastuzumab in brain lesions but not in normal brain, demonstrating ability of the antibody to penetrate the murine brain. No such uptake was seen with sham injections performed to rule out physical trauma of the procedure as the reason for access. In addition, uptake was equivalent in brain lesions compared to extracranial mammary fat pad tumors. It is unclear however, how the BBB or BTB may be affected or allow for more heterogenous access if brain lesions were formed by seeding from the systemic circulation akin to the normal course of the disease. Interestingly, despite equivalent uptake of trastuzumab, the authors also found that a 3 -fold higher systemic dose of trastuzumab was needed to achieve the same antitumor efficacy in brain lesions compared to mammary fat pad tumors. Proposed explanations for reduced efficacy in the brain were specific ligands in the brain microenvironment that mediate resistance to HER2directed drugs, or activation of downstream signaling such as $\mathrm{PI} 3 \mathrm{~K} / \mathrm{mTOR}$. The authors demonstrated enhanced 
survival when a brain-penetrant $\mathrm{PI} 3 \mathrm{~K} / \mathrm{mTOR}$ inhibitor was combined with trastuzumab, though it was unclear whether the effect was localized to the brain or impacted extracranial sites also. In another example, Stemmler et al. (59) showed increased trastuzumab levels in the CSF after WBRT (serum:csf 420:1 pre- vs. 76:1 post-radiation). Clinical data seems to support this effect. A CNS metastasis-focused analysis of the CLEOPATRA trial reported a significantly delayed onset of brain metastases in the pertuzumab arm by 3 months (61). An exploratory, retrospective analysis of the EMILIA trial data (62) demonstrated a significant improvement in OS in those patients with pre-existing CNS metastases receiving T-DM1 compared to the capecitabine/ lapatinib (OS $26.8 v$ vs. 12.9 months). In a study of the natural history of HER2 + BCBMs, Mounsey et al. (63) found that those who received HER2-targeted therapy (trastuzumab, lapatinib, pertuzumab or T-DM1) after diagnosis of brain metastases had an increased median OS of 2.1 years compared to 0.65 years for those who did not receive such targeted therapy, suggesting a direct effect in the brain. Greater control of tumor burden extra-cranially is also expected to decrease the risk of additional seeding to the CNS. Another approach to deliver monoclonal antibodies to the brain is directly via the intrathecal route, and case studies exist showing prolonged survival using this method in brain metastases and leptomeningeal carcinomatosis (64-67). A Phase I clinical trial of intrathecal pertuzumab and trastuzumab in patients with new, untreated, asymptomatic or low symptomatic HER2+ BCBM is now enrolling patients (NCT02598427).

A rational research approach to BCBMs is to look for agents that have systemic efficacy and sufficient brain penetration. Small, lipophilic molecules that could cross the BBB such as TKIs, with differing receptor selectivity, are attractive candidates. The degree to which the bloodtumor-barrier (BTB) is breached with metastases or prior radiation is unpredictable, however. Lapatinib, a reversible TKI of both HER1 and HER2 and one of the earliest TKIs studied in patients with progressive HER2+ BCBMs (68), had low response rate as monotherapy (2.6-6\%). In a subset of patients who crossed over to receive combination therapy with lapatinib plus cytotoxic capecitabine, response rates increased to approximately $20 \%$. This was confirmed in the phase II LANDSCAPE trial of front-line, combination lapatinib and capecitabine in WBRT-naïve HER2+ BCBM where a $67 \%$ response rate by volumetric measurements and a median time to progression of 5.5 months was observed (69). Lapatinib is a substrate for P-glycoprotein and breast cancer resistance protein efflux pumps in a murine model (70) and active efflux pumps inherent to the BTB may contribute to the lower than expected clinical response. As noted earlier, Tasker et al. in a murine model using ${ }^{14} \mathrm{C}$-lapatinib inhibitor (29), showed $>100$-fold differences in the distribution of ${ }^{14} \mathrm{C}$-lapatinib uptake both within and between brain metastases. The uptake was significantly correlated with permeability of the BTB and independent of size of the metastatic brain lesion. Moreover, analysis of tumor cells after treatment showed no evidence of lapatinib drug resistance. Morikawa et al. (71) demonstrated measurable concentrations of capecitabine and lapatinib in tissue samples of resected human BCBMs in 12 patients treated with the drug prior to surgery, thus providing evidence of $\mathrm{BBB} / \mathrm{BTB}$ penetration in actual patients. As with preclinical models, there was significant variability in drug uptake both between patients and within tumors. Such heterogeneity of BTB permeability and variable uptake of drug creates a challenge for drug delivery in treating BCBMs. A number of clinical trials are on-going combining lapatinib with chemotherapies, monoclonal antibodies and as a radiosensitizer for SRS or WBRT (see Table 1).

Neratinib, an irreversible TKI of HER1, HER2, and HER4, combined with paclitaxel was compared to trastuzumab-paclitaxel in the NEFERTT trial (72). While PFS of this combination was not superior to the trastuzumab-paclitaxel arm, a lower incidence of CNS metastases $(16.3 \%$ vs. $31.2 \%$, HR $=0.45)$ was seen and the Kaplan-Meier estimate of time to CNS disease was significantly longer in the neratinib arm, (not reached $v s$. 18.3 months). Results of the third cohort of the TBCRC 022 Phase II trial combining neratinib with capecitabine were presented at ASCO 2017 (73). The CNS overall response rate (ORR) was $49 \%$ by volumetric measurement, with median time to CNS progression of 5.5 months. Diarrhea is a major toxicity with neratinib, thus antidiarrheal prophylaxis and supportive care is paramount. The NALA phase III study comparing neratinib/capecitabine to lapatinib/capecitabine is currently enrolling patients, results anticipated (NCT01808573).

ONT-380 (tucatinib) is a reversible TKI that is highly selective to HER2 inhibition ( $\mathrm{IC}_{50}$ for HER2 of $8 \mathrm{nM} v$ s. $\mathrm{IC}_{50}$ for EGFR $>1,000 \mathrm{nM}$ ). A Phase Ib study combining ONT-380 with T-DM1 in patients with a median of 2 prior HER2 agents and those with brain metastases, showed an ORR of $47 \%$ and median PFS 8.2 months (74). Notably, the majority of adverse events were Grade 1 . A Phase Ib study of triplet therapy combining dual anti-HER2 with tucatinib 
Table 1 Selected on-going clinical trials for HER+ breast cancer brain metastases

\begin{tabular}{|c|c|c|c|c|}
\hline Class & Experimental arm & Control arm & Status & $\begin{array}{l}\text { ClinicalTrials.gov } \\
\text { identifier }\end{array}$ \\
\hline \multirow[t]{2}{*}{$\mathrm{mAb}$} & Intrathecal pertuzumab + trastuzumab & None (Phase I) & Recruiting & NCT02598427 \\
\hline & T-DM1 plus metronomic temozolomide & T-DM1 alone (Phase I/II) & Recruiting & NCT03190967 \\
\hline TKI & $\begin{array}{l}\text { Lapatinib (following trastuzumab and cranial } \\
\text { radiation) }\end{array}$ & None (Phase II) & $\begin{array}{l}\text { Active, not } \\
\text { recruiting }\end{array}$ & NCT00263588 \\
\hline $\mathrm{TKI}+$ radiation & Lapatinib + SRS or WBRT & SRS or WBRT & Recruiting & NCT01622868 \\
\hline \multirow[t]{2}{*}{$\begin{array}{l}\text { TKI + chemo- } \\
\text { therapy }\end{array}$} & Intermittent high-dose lapatinib + capecitabine & None (Phase I) & $\begin{array}{l}\text { Active, not } \\
\text { recruiting }\end{array}$ & NCT02650752 \\
\hline & $\begin{array}{l}\text { Tucatinib + capecitabine + trastuzumab } \\
\text { (HER2CLIMB trial) }\end{array}$ & $\begin{array}{l}\text { Placebo + capecitabine }+ \\
\text { trastuzumab }\end{array}$ & Recruiting & NCT02614794 \\
\hline $\begin{array}{l}\text { mTOR inhibitor + } \\
\text { chemotherapy }\end{array}$ & Everolimus + trastuzumab + vinorelbine & None (Phase II) & $\begin{array}{l}\text { Active, not } \\
\text { recruiting }\end{array}$ & NCT01305941 \\
\hline $\begin{array}{l}\text { PI3K inhibitor + } \\
\text { chemotherapy }\end{array}$ & $\begin{array}{l}\text { Buparlisib }(\text { BMK120) + capecitabine + } \\
\text { trastuzumab }\end{array}$ & None (single arm Phase II) & $\begin{array}{l}\text { Active, not } \\
\text { recruiting }\end{array}$ & NCT02000882 \\
\hline CDK4/6 & Palbociclib +/- trastuzumab & None (single arm Phase II) & Recruiting & NCT02774681 \\
\hline
\end{tabular}

mAb, monoclonal antibody; TKI, tyrosine kinase inhibitor; mTOR, mammalian target of rapamycin (a kinase); PI3K, phosphatidylinositol3-kinase; CDK, cyclin-dependent kinase; BCBM, brain cancer brain metastases; SRS, stereotactic radiosurgery; WBRT, whole brain radiotherapy.

and trastuzumab plus cytotoxic capecitabine in a cohort of 23 patients ( $40 \%$ had baseline brain metastases) had an ORR $61 \%$ and median duration of response of 10 months (95\% CI, 2.8-19.3). Median PFS was 7.8 months (95\% CI, 4.1-12.4). Outcomes for those with pre-existing brain metastases were similar to those without (75). Based on these positive data, the Phase 2 HER2CLIMB trial of this triplet combination is currently recruiting (NCT02614794).

Inhibitors of pathways downstream from HER2, such as mTOR, PI3K and CDK4/6, are also being investigated. A selection of ongoing clinical trials with a variety of different experimental agents with brain penetration addressing HER2+ BCBMs are shown in Table 1.

\section{Hormone receptor-positive disease}

CNS metastases occur less frequently and later in the disease for HR+/HER2- breast cancer compared to other subtypes. Activation of the phosphoinositide 3 kinase (PI3K)/Akt/mammalian target of rapamycin (mTOR) pathway causes resistance to endocrine therapy for these patients (76-80). The BOLERO-2 trial $(81,82)$ led to approval of the mTOR inhibitor, everolimus, combined with an endocrine agent, to overcome this resistance, but patients with brain metastases were excluded. The BOLERO-6 trial comparing everolimus and capecitabine is ongoing (NCT01783444). PIK3CA activating mutations are reported to occur in $28-47 \%$ of $\mathrm{HR}+$ disease $(79,83)$. In a preclinical model, Chen et al. showed that PI3K inhibition with buparlisib (a pan-PI3K inhibitor) and alpelisib (a selective PI3K $\alpha$ inhibitor) sensitized ER+ BC cell lines to tamoxifen (84). Le Rhun et al. found that single nucleotide polymorphisms in the PI3KR1 gene were associated with CNS metastases (85). Several trials are on-going exploring 
Table 2 Selected on-going clinical trials for HR+ breast cancer

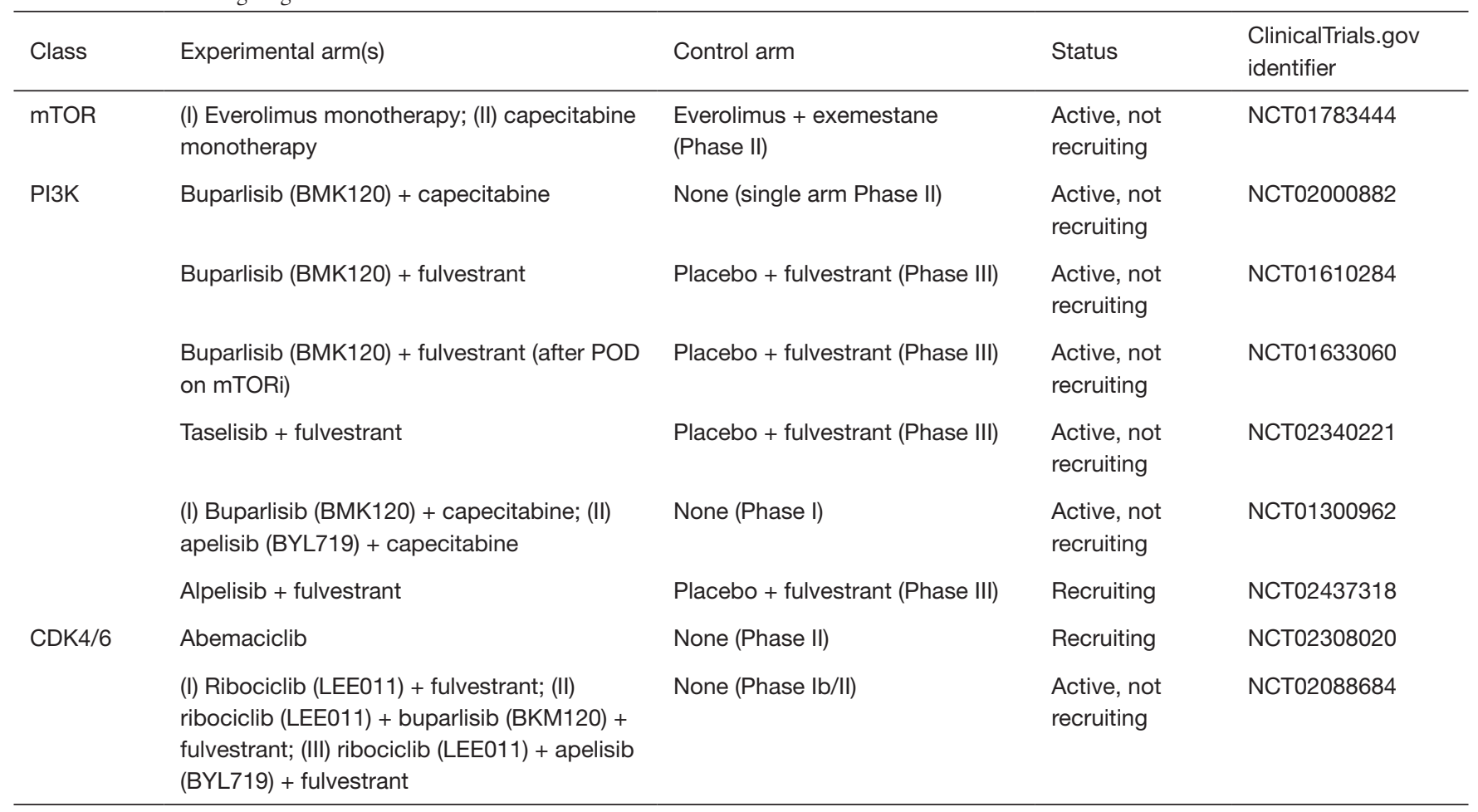

Mtor, mammalian target of rapamycin (a kinase); PI3K, phosphatidylinositol-3-kinase; CDK, cyclin-dependent kinase; POD, progression of disease.

efficacy of several PI3K inhibitors. The STAR trial with buparlisib (NCT02000882) is specifically for brain metastases, while trials with alpelisib and taselisib, both $\mathrm{PI} 3 \mathrm{~K} \alpha$ inhibitors, are for systemic treatment of MBC. In the latter two trials, patients with BCBMs are included as long as they do not have symptomatic brain metastases (see Table 2).

Growth of HR+ BC depends on cyclin D1, a transcriptional target of the endocrine receptor, which then activates cyclin-dependent kinases 4 and 6 (CDK 4/6) causing G1-S phase transition and cell cycle entry. Two CDK 4/6 inhibitors combined with endocrine therapy prolong PFS and are FDA approved in the first line setting: palbociclib $(86,87)$ and ribociclib $(88)$. Another CDK 4/6 inhibitor, abemaciclib, received FDA approval in 2017 combined with fulvestrant for use in patients who had progressed on endocrine therapy alone (89) and as monotherapy for heavily pretreated patients with refractory $\mathrm{HR}^{+} / \mathrm{HER} 2^{-}$disease (90). Interim results (91) of the phase III MONARCH-3 study (NCT02246621) with abemaciclib in first line setting, also show prolonged PFS (not reached vs. 14.7 months in placebo arm, HR $=0.54$ ). OS data are not yet mature. Abemaciclib has more selectivity for CDK4 over CDK6 and, importantly, compared to palbociclib and ribociclib, has shown ability to penetrate the CNS in preclinical model (92). A phase I trial in patients with breast, non-small cell lung cancer (NSCLC), glioblastoma, melanoma, and colorectal cancers, showed abemaciclib concentrations in the CSF approximating that in plasma $(93,94)$. Preliminary interim analysis $(95)$ of the ongoing phase II trial targeting brain metastases with abemaciclib (NCT02308020) has demonstrated partial responses in the CNS in two HR+ breast cancer patients.

\section{TNBC}

Almost half of patients with advanced TNBC develop brain metastases with inferior survival compared to non-TNBC subtypes and new treatment options are urgently needed. The backbone of systemic treatment has been platinum or taxane-based chemotherapies, though patients with BCBMs were not included in these original trials. Patients with BRCA1 germline mutations are known to develop TNBC (96) and approximately $20 \%$ of TNBCs harbor a $B R C A 1 / 2$ 
Table 3 Selected on-going clinical trials for triple negative breast cancer

\begin{tabular}{|c|c|c|c|c|}
\hline Class & Experimental arm(s) & Control arm & Status & $\begin{array}{l}\text { ClinicalTrials.gov } \\
\text { identifier }\end{array}$ \\
\hline \multirow[t]{4}{*}{ PARP } & $\begin{array}{l}\text { (I) Olaparib + carboplatin; } \\
\text { (II) olaparib + paclitaxel; } \\
\text { (III) olaparib + carboplatin + paclitaxel }\end{array}$ & None (Phase I) & $\begin{array}{l}\text { Active, not } \\
\text { recruiting }\end{array}$ & NCT00516724 \\
\hline & $\begin{array}{l}\text { Talazoparib }(\mathrm{BMN}-673)+\text { carboplatin }+ \\
\text { paclitaxel }\end{array}$ & None (Phase I) & $\begin{array}{l}\text { Active, not } \\
\text { recruiting }\end{array}$ & NCT02358200 \\
\hline & Cisplatin + veliparib & Cisplatin + placebo (Phase II) & Recruiting & NCT02595905 \\
\hline & $\begin{array}{l}\text { Rucaparib (in patients with a BRCAness } \\
\text { genomic signature) }\end{array}$ & None (Phase II) & Recruiting & NCT02505048 \\
\hline \multirow{3}{*}{$\mathrm{mAb} \mathrm{l} / \mathrm{O}$} & $\begin{array}{l}\text { Atezolizumab + pegylated liposomal } \\
\text { doxorubicin + cyclophosphamide }\end{array}$ & $\begin{array}{l}\text { Placebo + pegylated liposomal } \\
\text { doxorubicin + cyclophosphamide }\end{array}$ & Recruiting & NCT03164993 \\
\hline & Pembrolizumab monotherapy & $\begin{array}{l}\text { Physician's choice: capecitabine, eribulin, } \\
\text { gemcitabine, or vinorelbine (Phase III) }\end{array}$ & $\begin{array}{l}\text { Active, not } \\
\text { recruiting }\end{array}$ & NCT02555657 \\
\hline & $\begin{array}{l}\text { (I) Pembrolizumab + nab-paclitaxel; } \\
\text { (II) Pembrolizumab + paclitaxel; } \\
\text { (III) Pembrolizumab + gemcitabine/ } \\
\text { carboplatin }\end{array}$ & $\begin{array}{l}\text { (I) Placebo + nab-paclitaxel; (II) placebo } \\
\text { + paclitaxel; (III) placebo + gemcitabine/ } \\
\text { carboplatin (Phase III) }\end{array}$ & Recruiting & NCT02819518 \\
\hline
\end{tabular}

PARP, poly(ADP-ribose) polymerase; mAb, monoclonal antibody; I/O, immunotherapy.

mutation (97). A recent next generation sequencing study of gene alterations in stage II-IV sporadic TNBC showed that the most frequently mutated genes were $T P 53, K D R$, PIK3CA, ATM, AKT1 and KIT and that along with BRCA1/2 mutations, these were correlated with a poor prognosis (98).

Agents that prevent repair of DNA such as poly (ADP-ribose) polymerase (PARP) inhibitors have been evaluated in TNBC $(99,100)$. The OlympiAD phase III trial (101) comparing the oral PARP inhibitor olaparib to single agent chemotherapy (capecitabine, vinorelbine, or eribulin) in TNBC with germline BRCA mutation showed improved PFS for the targeted agent (7.0 vs. 4.2 months; $\mathrm{HR}=0.58)$. Karginova $e t$ al. demonstrated brain penetrance of the PARP inhibitor veliparib in $B R C A$-mutant TNBC intracranial murine models with a survival benefit when combined with carboplatin $(102,103)$. A Phase II trial, specifically including a brain metastases cohort, is currently recruiting comparing cisplatin combined with veliparib to cisplatin monotherapy in TNBC +/- BRCA mutation
(NCT02595905). Additional studies are underway with different PARP inhibitors combined with chemotherapy (see Table 3). A trial of Eribulin, a microtubule inhibitor already FDA approved in MBC, is currently recruiting for a phase II study exploring its efficacy in treatment of brain metastases (NCT02581839).

TNBC is the most immunogenic of the subtypes making checkpoint inhibition of programmed cell death protein 1 receptor (PD-1) and programmed cell death protein 1 receptor ligand (PD-L1) attractive therapeutic targets. In the metastatic setting, the phase 1b KEYNOTE-012 trial (104) of pembrolizumab (a monoclonal antibody that binds to the PD-1 receptor and blocks its interaction with PD-L1), the ORR was $18.5 \%$ with a median time to response of 17.9 weeks. Similarly, PD-L1 inhibitors atezolizumab (105) and avelumab (106) have also shown activity in TNBC. In the phase I trial with atezolizumab (105), ORR was $10-13 \%$ and median OS was 9.3 months in all patients. One-year overall survival was $41 \%$, and $22 \%$ at 2 years. 
Exploratory biomarker analysis suggested that higher response rates seemed to be associated with higher levels of TILs and higher levels of CD8 T cells (107). A number of trials with immunotherapy as single agent or combined with chemotherapy to stimulate the immune response are on-going in early stage and metastatic TNBC, but none has yet to specifically target BCBMs (sample trials shown in Table 3). In a retrospective study of 84 brain metastases, Duchnowska et al. showed PD-L1 and PD-L2 expression in $\mathrm{BCBM}$ was present in $53 \%$ and $36 \%$ of cases, and $\mathrm{PD}-1$ expression on TILs correlated positively with CD4+ and CD8+ TILs (108). While hopeful, it remains to be seen if immunotherapy also offers efficacy in BCBMs.

\section{Conclusions}

The landscape for managing $\mathrm{BCBM}$ is improving significantly, due in large part due to a better understanding of the biology of brain metastases leading to more-precisely targeted options. Immunotherapy offers promise, though as seen in extracranial disease, suitable biomarkers remain elusive to predict likely responders. Novel drug delivery systems such as nanoparticles that can cross the BBB to deliver cytotoxic are also being explored. While there are as yet no FDA approved treatments for BCBMs, many clinical trials now allow patients with stable and progressive brain metastases to enroll, opening up opportunities to find new signals to pursue and possible immediate benefit to the patients on these trials.

\section{Acknowledgements}

None.

\section{Footnote}

Conflicts of Interest: CK Anders, has consulted for Novartis, Sanofi, toBBB, GERON, Angiochem, Merrimack, Lily, Genentech, Nektar, and Kadmon and has received funding from Novartis, Sanofi, toBBB, GERON, Angiochem, Merrimack, PUMA, Lily, Merck, Oncothyreon, Cascadian, Nektar and Tesaro. EM Brosnan has no conflicts of interest to declare.

\section{References}

1. Mayer M. A patient perspective on brain metastases in breast cancer. Clin Cancer Res 2007;13:1623-4.

2. Barnholtz-Sloan JS, Sloan AE, Davis FG, et al. Incidence proportions of brain metastases in patients diagnosed (1973 to 2001) in the Metropolitan Detroit Cancer Surveillance System. J Clin Oncol 2004;22:2865-72.

3. Lin NU, Bellon JR, Winer EP. CNS metastases in breast cancer. J Clin Oncol 2004;22:3608-17.

4. Tsukada Y, Fouad A, Pickren JW, et al. Central nervous system metastasis from breast carcinoma. Autopsy study. Cancer 1983;52:2349-54.

5. Aversa C, Rossi V, Geuna E, et al. Metastatic breast cancer subtypes and central nervous system metastases. Breast 2014:23:623-8.

6. Niikura N, Hayashi N, Masuda N, et al. Treatment outcomes and prognostic factors for patients with brain metastases from breast cancer of each subtype: a multicenter retrospective analysis. Breast Cancer Res Treat 2014;147:103-12.

7. Sperduto PW, Kased N, Roberge D, et al. The effect of tumor subtype on the time from primary diagnosis to development of brain metastases and survival in patients with breast cancer. J Neurooncol 2013;112:467-72.

8. Bendell JC, Domchek SM, Burstein HJ, et al. Central nervous system metastases in women who receive trastuzumab-based therapy for metastatic breast carcinoma. Cancer 2003;97:2972-7.

9. Pestalozzi BC, Holmes E, de Azambuja E, et al. CNS relapses in patients with HER2-positive early breast cancer who have and have not received adjuvant trastuzumab: a retrospective substudy of the HERA trial (BIG 1-01). Lancet Oncol 2013;14:244-8.

10. Lin NU, Claus E, Sohl J, et al. Sites of distant recurrence and clinical outcomes in patients with metastatic triplenegative breast cancer: high incidence of central nervous system metastases. Cancer 2008;113:2638-45.

11. Kaidar-Person O, Meattini I, Jain P, et al. Discrepancies between biomarkers of primary breast cancer and subsequent brain metastases: an international multicenter study. Breast Cancer Res Treat 2018;167:479-83.

12. Priedigkeit N, Hartmaier RJ, Chen Y, et al. Intrinsic Subtype Switching and Acquired ERBB2/HER2 Amplifications and Mutations in Breast Cancer Brain Metastases. JAMA Oncol 2017;3:666-71.

13. Deeken JF, Loscher W. The blood-brain barrier and cancer: transporters, treatment, and Trojan horses. Clin Cancer Res 2007;13:1663-74.

14. Fong CW. Permeability of the Blood-Brain Barrier: Molecular Mechanism of Transport of Drugs and Physiologically Important Compounds. J Membr Biol 2015;248:651-69. 
15. Linskey ME, Andrews DW, Asher AL, et al. The role of stereotactic radiosurgery in the management of patients with newly diagnosed brain metastases: a systematic review and evidence-based clinical practice guideline. $\mathrm{J}$ Neurooncol 2010;96:45-68.

16. Pardridge WM. Brain Drug Targeting: The Future of Brain Drug Development. Cambridge, UK: Cambridge University Press, 2001.

17. Eyal S, Hsiao P, Unadkat JD. Drug interactions at the blood-brain barrier: fact or fantasy? Pharmacol Ther 2009;123:80-104.

18. Mehta MP, Paleologos NA, Mikkelsen T, et al. The role of chemotherapy in the management of newly diagnosed brain metastases: a systematic review and evidence-based clinical practice guideline. J Neurooncol 2010;96:71-83.

19. Wilhelm I, Molnar J, Fazakas C, et al. Role of the bloodbrain barrier in the formation of brain metastases. Int $\mathrm{J}$ Mol Sci 2013;14:1383-411.

20. Gabathuler R. Approaches to transport therapeutic drugs across the blood-brain barrier to treat brain diseases. Neurobiol Dis 2010;37:48-57.

21. Lipinski CA, Lombardo F, Dominy BW, et al. Experimental and computational approaches to estimate solubility and permeability in drug discovery and development settings. Adv Drug Deliv Rev 2001;46:3-26.

22. Muldoon LL, Soussain C, Jahnke K, et al. Chemotherapy delivery issues in central nervous system malignancy: a reality check. J Clin Oncol 2007;25:2295-305.

23. Kemper EM, van Zandbergen AE, Cleypool C, et al. Increased penetration of paclitaxel into the brain by inhibition of P-Glycoprotein. Clin Cancer Res 2003;9:2849-55.

24. Elsinga PH, Hendrikse NH, Bart J, et al. PET Studies on $\mathrm{P}$-glycoprotein function in the blood-brain barrier: how it affects uptake and binding of drugs within the CNS. Curr Pharm Des 2004;10:1493-503.

25. Rosner D, Nemoto T, Lane WW. Chemotherapy induces regression of brain metastases in breast carcinoma. Cancer 1986;58:832-9.

26. Bart J, Groen HJ, Hendrikse NH, et al. The blood-brain barrier and oncology: new insights into function and modulation. Cancer Treat Rev 2000;26:449-62.

27. Regina A, Demeule M, Laplante A, et al. Multidrug resistance in brain tumors: roles of the blood-brain barrier. Cancer Metastasis Rev 2001;20:13-25.

28. Lockman PR, Mittapalli RK, Taskar KS, et al. Heterogeneous blood-tumor barrier permeability determines drug efficacy in experimental brain metastases of breast cancer. Clin Cancer Res 2010;16:5664-78.

29. Taskar KS, Rudraraju V, Mittapalli RK, et al. Lapatinib distribution in HER2 overexpressing experimental brain metastases of breast cancer. Pharm Res 2012;29:770-81.

30. Adkins CE, Mittapalli RK, Manda VK, et al. P-glycoprotein mediated efflux limits substrate and drug uptake in a preclinical brain metastases of breast cancer model. Front Pharmacol 2013;4:136.

31. Bos PD, Zhang XH, Nadal C, et al. Genes that mediate breast cancer metastasis to the brain. Nature 2009;459:1005-9.

32. Drolez A, Vandenhaute E, Delannoy CP, et al. ST6GALNAC5 Expression Decreases the Interactions between Breast Cancer Cells and the Human Blood-Brain Barrier. Int J Mol Sci 2016;17.

33. Avraham HK, Jiang S, Fu Y, et al. Angiopoietin-2 mediates blood-brain barrier impairment and colonization of triple-negative breast cancer cells in brain. J Pathol 2014;232:369-81.

34. Yonemori K, Tsuta K, Ono M, et al. Disruption of the blood brain barrier by brain metastases of triple-negative and basal-type breast cancer but not HER2/neu-positive breast cancer. Cancer 2010;116:302-8.

35. Palmieri D, Bronder JL, Herring JM, et al. Her-2 overexpression increases the metastatic outgrowth of breast cancer cells in the brain. Cancer Res 2007;67:4190-8.

36. Da Silva L, Simpson PT, Smart CE, et al. HER3 and downstream pathways are involved in colonization of brain metastases from breast cancer. Breast Cancer Res 2010;12:R46.

37. Salhia B, Kiefer J, Ross JT, et al. Integrated genomic and epigenomic analysis of breast cancer brain metastasis. PLoS One 2014;9:e85448.

38. Neman J, Termini J, Wilczynski S, et al. Human breast cancer metastases to the brain display GABAergic properties in the neural niche. Proc Natl Acad Sci U S A 2014;111:984-9.

39. Kim SJ, Kim JS, Park ES, et al. Astrocytes upregulate survival genes in tumor cells and induce protection from chemotherapy. Neoplasia 2011;13:286-98.

40. Zhang L, Zhang S, Yao J, et al. Microenvironmentinduced PTEN loss by exosomal microRNA primes brain metastasis outgrowth. Nature 2015;527:100-4.

41. Hohensee I, Chuang HN, Grottke A, et al. PTEN mediates the cross talk between breast and glial cells in brain metastases leading to rapid disease progression. Oncotarget 2017;8:6155-68.

42. Narloch J, Luedke C, Broadwater G, et al. Number of 
tumor-infiltrating lymphocytes in breast cancer brain metastases compared to matched breast primaries. J Clin Oncol 2017;35:abstr 2049.

43. Ogiya R, Niikura N, Kumaki N, et al. Immune microenvironment in brain metastases of breast cancer. J Clin Oncol 2017;35:abstr 1081.

44. Prince G, Deal AM, McKee MJ, et al. Examination and prognostic implications of the unique microenvironment of breast cancer brain metastases. J Clin Oncol suppl 2017;suppl_15;abstr 2072.

45. Patchell RA, Tibbs PA, Walsh JW, et al. A randomized trial of surgery in the treatment of single metastases to the brain. N Engl J Med 1990;322:494-500.

46. Andrews DW, Scott CB, Sperduto PW, et al. Whole brain radiation therapy with or without stereotactic radiosurgery boost for patients with one to three brain metastases: phase III results of the RTOG 9508 randomised trial. Lancet 2004;363:1665-72.

47. Chang EL, Wefel JS, Hess KR, et al. Neurocognition in patients with brain metastases treated with radiosurgery or radiosurgery plus whole-brain irradiation: a randomised controlled trial. Lancet Oncol 2009;10:1037-44.

48. Brown PD, Jaeckle K, Ballman KV, et al. Effect of Radiosurgery Alone vs Radiosurgery With Whole Brain Radiation Therapy on Cognitive Function in Patients With 1 to 3 Brain Metastases: A Randomized Clinical Trial. JAMA 2016;316:401-9.

49. Tsao MN, Lloyd NS, Wong RK, et al. Radiotherapeutic management of brain metastases: a systematic review and meta-analysis. Cancer Treat Rev 2005;31:256-73.

50. Viani GA, Manta GB, Fonseca EC, et al. Whole brain radiotherapy with radiosensitizer for brain metastases. J Exp Clin Cancer Res 2009;28:1.

51. McArthur H, Beal K, Halpenny D, et al. CTLA4 blockade with HER2-directed therapy $(\mathrm{H})$ yields clinical benefit in women undergoing radiation therapy (RT) for HER2positive (HER2+) breast cancer brain metastases (BCBM). Cancer Res 2017;77:4705.

52. Gondi V, Pugh SL, Tome WA, et al. Preservation of memory with conformal avoidance of the hippocampal neural stem-cell compartment during whole-brain radiotherapy for brain metastases (RTOG 0933): a phase II multi-institutional trial. J Clin Oncol 2014;32:3810-6.

53. Wu SG, Sun JY, Tong Q, et al. Clinical features of brain metastases in breast cancer: an implication for hippocampal-sparing whole-brain radiation therapy. Ther Clin Risk Manag 2016;12:1849-53.

54. Brown PD, Pugh S, Laack NN, et al. Memantine for the prevention of cognitive dysfunction in patients receiving whole-brain radiotherapy: a randomized, double-blind, placebo-controlled trial. Neuro Oncol 2013;15:1429-37.

55. Kirson ED, Dbaly V, Tovarys F, et al. Alternating electric fields arrest cell proliferation in animal tumor models and human brain tumors. Proc Natl Acad Sci U S A 2007;104:10152-7.

56. Kirson ED, Schneiderman RS, Dbaly V, et al. Chemotherapeutic treatment efficacy and sensitivity are increased by adjuvant alternating electric fields (TTFields). BMC Med Phys 2009;9:1.

57. Stupp R, Taillibert S, Kanner AA, et al. Maintenance Therapy With Tumor-Treating Fields Plus Temozolomide vs Temozolomide Alone for Glioblastoma: A Randomized Clinical Trial. JAMA 2015;314:2535-43.

58. Dijkers EC, Oude Munnink TH, Kosterink JG, et al. Biodistribution of $89 \mathrm{Zr}$-trastuzumab and PET imaging of HER2-positive lesions in patients with metastatic breast cancer. Clin Pharmacol Ther 2010;87:586-92.

59. Stemmler HJ, Schmitt M, Willems A, et al. Ratio of trastuzumab levels in serum and cerebrospinal fluid is altered in HER2-positive breast cancer patients with brain metastases and impairment of blood-brain barrier. Anticancer Drugs 2007;18:23-8.

60. Lewis Phillips GD, Nishimura MC, Lacap JA, et al. Trastuzumab uptake and its relation to efficacy in an animal model of HER2-positive breast cancer brain metastasis. Breast Cancer Res Treat 2017;164:581-91.

61. Swain SM, Baselga J, Miles D, et al. Incidence of central nervous system metastases in patients with HER2positive metastatic breast cancer treated with pertuzumab, trastuzumab, and docetaxel: results from the randomized phase III study CLEOPATRA. Ann Oncol 2014;25:1116-21.

62. Krop IE, Lin NU, Blackwell K, et al. Trastuzumab emtansine (T-DM1) versus lapatinib plus capecitabine in patients with HER2-positive metastatic breast cancer and central nervous system metastases: a retrospective, exploratory analysis in EMILIA. Ann Oncol 2015;26:113-9.

63. Mounsey LA, Deal AM, Keith KC, et al. Changing Natural History of HER2-Positive Breast Cancer Metastatic to the Brain in the Era of New Targeted Therapies. Clin Breast Cancer 2018;18:29-37.

64. Bousquet G, Darrouzain F, de Bazelaire C, et al. Intrathecal Trastuzumab Halts Progression of CNS Metastases in Breast Cancer. J Clin Oncol 2016;34:e151-5.

65. Pluchart H, Jacquet E, Charlety D, et al. Long-Term Survivor with Intrathecal and Intravenous Trastuzumab Treatment in Metastatic Breast Cancer. Target Oncol 
2016;11:687-91.

66. Stemmler HJ, Schmitt M, Harbeck N, et al. Application of intrathecal trastuzumab (Herceptintrade mark) for treatment of meningeal carcinomatosis in HER2-overexpressing metastatic breast cancer. Oncol Rep 2006;15:1373-7.

67. Zagouri F, Sergentanis TN, Bartsch R, et al. Intrathecal administration of trastuzumab for the treatment of meningeal carcinomatosis in HER2-positive metastatic breast cancer: a systematic review and pooled analysis. Breast Cancer Res Treat 2013;139:13-22.

68. Lin NU, Carey LA, Liu MC, et al. Phase II trial of lapatinib for brain metastases in patients with human epidermal growth factor receptor 2-positive breast cancer. J Clin Oncol 2008;26:1993-9.

69. Bachelot T, Romieu G, Campone M, et al. Lapatinib plus capecitabine in patients with previously untreated brain metastases from HER2-positive metastatic breast cancer (LANDSCAPE): a single-group phase 2 study. Lancet Oncol 2013;14:64-71.

70. Polli JW, Olson KL, Chism JP, et al. An unexpected synergist role of $\mathrm{P}$-glycoprotein and breast cancer resistance protein on the central nervous system penetration of the tyrosine kinase inhibitor lapatinib (N-\{3-chloro-4-[(3fluorobenzyl)oxy]phenyl\}-6-[5-(\{[2-(methylsulfonyl)ethyl] amino \}methyl)-2-furyl]-4-quinazolinamine; GW572016). Drug Metab Dispos 2009;37:439-42.

71. Morikawa A, Peereboom DM, Thorsheim HR, et al. Capecitabine and lapatinib uptake in surgically resected brain metastases from metastatic breast cancer patients: a prospective study. Neuro Oncol 2015;17:289-95.

72. Awada A, Colomer R, Inoue K, et al. Neratinib Plus Paclitaxel vs Trastuzumab Plus Paclitaxel in Previously Untreated Metastatic ERBB2-Positive Breast Cancer: The NEfERT-T Randomized Clinical Trial. JAMA Oncol 2016;2:1557-64.

73. Freedman RA, Gelman RS, Melisko ME, et al. TBCRC 022: Phase II trial of neratinib for patients (Pts) with human epidermal growth factor receptor 2 (HER2+) breast cancer and brain metastases (BCBM). J Clin Oncol 2014;32:1005.

74. Borges VF, Ferrario C, Aucoin N, et al. Efficacy results of a phase $1 \mathrm{~b}$ study of ONT-380, a CNS-penetrant TKI, in combination with T-DM1 in HER2+ metastatic breast cancer (MBC), including patients (pts) with brain metastases. J Clin Oncol 2016;34:513.

75. Hamilton E, Borges V, Conlin A, et al. Efficacy results of a phase 1b study of ONT-380, an oral HER2-specific inhibitor, in combination with capecitabine (C) and trastuzumab (T) in HER2 + metastatic breast cancer (MBC), including patients (pts) with brain metastases (mets). Cancer Res 2017;77.

76. Crowder RJ, Phommaly C, Tao Y, et al. PIK3CA and PIK3CB inhibition produce synthetic lethality when combined with estrogen deprivation in estrogen receptorpositive breast cancer. Cancer Res 2009;69:3955-62.

77. Johnston SR. Clinical efforts to combine endocrine agents with targeted therapies against epidermal growth factor receptor/human epidermal growth factor receptor 2 and mammalian target of rapamycin in breast cancer. Clin Cancer Res 2006;12:1061s-8s.

78. Ellis MJ, Perou CM. The genomic landscape of breast cancer as a therapeutic roadmap. Cancer Discov 2013;3:27-34.

79. Lee JJ, Loh K, Yap YS. PI3K/Akt/mTOR inhibitors in breast cancer. Cancer Biol Med 2015;12:342-54.

80. Miller TW, Hennessy BT, Gonzalez-Angulo AM, et al. Hyperactivation of phosphatidylinositol-3 kinase promotes escape from hormone dependence in estrogen receptor-positive human breast cancer. J Clin Invest 2010;120:2406-13.

81. Baselga J, Campone M, Piccart M, et al. Everolimus in postmenopausal hormone-receptor-positive advanced breast cancer. N Engl J Med 2012;366:520-9.

82. Piccart M, Hortobagyi GN, Campone M, et al. Everolimus plus exemestane for hormone-receptor-positive, human epidermal growth factor receptor-2-negative advanced breast cancer: overall survival results from BOLERO2dagger. Ann Oncol 2014;25:2357-62.

83. Stemke-Hale K, Gonzalez-Angulo AM, Lluch A, et al. An integrative genomic and proteomic analysis of PIK3CA, PTEN, and AKT mutations in breast cancer. Cancer Res 2008;68:6084-91.

84. Chen IC, Hsiao LP, Huang IW, et al. Phosphatidylinositol-3 Kinase Inhibitors, Buparlisib and Alpelisib, Sensitize Estrogen Receptor-positive Breast Cancer Cells to Tamoxifen. Sci Rep 2017;7:9842.

85. Le Rhun E, Bertrand N, Dumont A, et al. Identification of single nucleotide polymorphisms of the PI3K-AKTmTOR pathway as a risk factor of central nervous system metastasis in metastatic breast cancer. Eur J Cancer 2017;87:189-98.

86. Finn RS, Martin M, Rugo HS, et al. Palbociclib and Letrozole in Advanced Breast Cancer. N Engl J Med 2016;375:1925-36.

87. Turner NC, Ro J, Andre F, et al. Palbociclib in HormoneReceptor-Positive Advanced Breast Cancer. N Engl J Med 2015;373:209-19. 
88. Hortobagyi GN, Stemmer SM, Burris HA, et al. Ribociclib as First-Line Therapy for HR-Positive, Advanced Breast Cancer. N Engl J Med 2016;375:1738-48.

89. Sledge GW Jr, Toi M, Neven P, et al. MONARCH 2: Abemaciclib in Combination With Fulvestrant in Women With HR+/HER2- Advanced Breast Cancer Who Had Progressed While Receiving Endocrine Therapy. J Clin Oncol 2017;35:2875-84.

90. Dickler MN, Tolaney SM, Rugo HS, et al. MONARCH 1, A Phase II Study of Abemaciclib, a CDK4 and CDK6 Inhibitor, as a Single Agent, in Patients with Refractory HR(+)/HER2(-) Metastatic Breast Cancer. Clin Cancer Res 2017;23:5218-24.

91. Goetz MP, Toi M, Campone M, et al. MONARCH 3: Abemaciclib As Initial Therapy for Advanced Breast Cancer. J Clin Oncol 2017;35:3638-46.

92. Raub TJ, Wishart GN, Kulanthaivel P, et al. Brain Exposure of Two Selective Dual CDK4 and CDK6 Inhibitors and the Antitumor Activity of CDK4 and CDK6 Inhibition in Combination with Temozolomide in an Intracranial Glioblastoma Xenograft. Drug Metab Dispos 2015;43:1360-71.

93. Patnaik A, Rosen LS, Tolaney SM, et al. Efficacy and Safety of Abemaciclib, an Inhibitor of CDK4 and CDK6, for Patients with Breast Cancer, Non-Small Cell Lung Cancer, and Other Solid Tumors. Cancer Discov 2016;6:740-53.

94. Sahebjam S, Le Rhun E, Kulanthaivel P, et al. Assessment of concentrations of abemaciclib and its major active metabolites in plasma, CSF, and brain tumor tissue in patients with brain metastases secondary to hormone receptor positive (HR+) breast cancer. J Clin Oncol 2016;34:abstr 526.

95. Tolaney SM, Lin NU, Thorton D, et al. Abemaciclib for the treatment of brain metastases (BM) secondary to hormone receptor positive (HR+), HER2 negative breast cancer. J Clin Oncol 2017;35:abstr 1019.

96. Atchley DP, Albarracin CT, Lopez A, et al. Clinical and pathologic characteristics of patients with BRCApositive and BRCA-negative breast cancer. J Clin Oncol 2008;26:4282-8.

97. Gonzalez-Angulo AM, Timms KM, Liu S, et al. Incidence and outcome of BRCA mutations in unselected patients with triple receptor-negative breast cancer. Clin Cancer Res 2011;17:1082-9.

98. Pop LA, Cojocneanu-Petric RM, Pileczki V, et al. Genetic alterations in sporadic triple negative breast cancer. Breast 2018;38:30-8.
99. Gelmon KA, Tischkowitz M, Mackay H, et al. Olaparib in patients with recurrent high-grade serous or poorly differentiated ovarian carcinoma or triple-negative breast cancer: a phase 2, multicentre, open-label, non-randomised study. Lancet Oncol 2011;12:852-61.

100. Tutt A, Robson M, Garber JE, et al. Oral poly(ADPribose) polymerase inhibitor olaparib in patients with BRCA1 or BRCA2 mutations and advanced breast cancer: a proof-of-concept trial. Lancet 2010;376:235-44.

101. Robson M, Im SA, Senkus E, et al. Olaparib for Metastatic Breast Cancer in Patients with a Germline BRCA Mutation. N Engl J Med 2017;377:523-33.

102. Donawho CK, Luo Y, Luo Y, et al. ABT-888, an orally active poly(ADP-ribose) polymerase inhibitor that potentiates DNA-damaging agents in preclinical tumor models. Clin Cancer Res 2007;13:2728-37.

103. Karginova O, Siegel MB, Van Swearingen AE, et al. Efficacy of Carboplatin Alone and in Combination with ABT888 in Intracranial Murine Models of BRCA-Mutated and BRCA-Wild-Type Triple-Negative Breast Cancer. Mol Cancer Ther 2015;14:920-30.

104. Nanda R, Chow LQ, Dees EC, et al. Pembrolizumab in Patients With Advanced Triple-Negative Breast Cancer: Phase Ib KEYNOTE-012 Study. J Clin Oncol 2016;34:2460-7.

105. Emens LA, Braiteh FS, Cassier P, et al. Inhibition of PDL1 by MPDL3280A leads to clinical activity in patients with metastatic triple-negative breast cancer. Cancer Res 2014;75:abstract nr PD1-6.

106. Dirix L, Takacs I, Nikolinakos P, et al. Avelumab (MSB0010718C), an anti-PD-L1 antibody, in patients with locally advanced or metastatic breast cancer: A phase Ib JAVELIN solid tumor trial. Cancer Res 2015;76.

107.Schmid P, Cruz C, Braiteh FS, et al. Atezolizumab in metastatic TNBC (mTNBC): Long-term clinical outcomes and biomarker analyses. Cancer Res 2017;77:abstract nr 2986.

108. Duchnowska R, Peksa R, Radecka B, et al. Immune response in breast cancer brain metastases and their microenvironment: the role of the PD-1/PD-L axis. Breast Cancer Res 2016;18:43.

Cite this article as: Brosnan EM, Anders CK. Understanding patterns of brain metastasis in breast cancer and designing rational therapeutic strategies. Ann Transl Med 2018;6(9):163. doi: 10.21037/atm.2018.04.35 